\title{
Topological evolution of surface transportation networks
}

\author{
Feng Xie ${ }^{\mathrm{a}, *}$, David Levinson ${ }^{\mathrm{b}}$ \\ a University of Minnesota, Department of Civil Engineering, Minneapolis, MN 55455, USA \\ b University of Minnesota, Department of Civil Engineering, 500 Pillsbury Drive SE, Minneapolis, MN 55455, USA
}

\section{A R T I C L E I N F O}

\section{Article history:}

Received 18 December 2007

Received in revised form 16 September

2008

Accepted 17 September 2008

\section{Keywords:}

Surface transportation network

Evolution

Simulation

Degeneration

Topology

\begin{abstract}
A B S T R A C T
This study explores the topological evolution of surface transportation networks, using empirical evidence and a simulation model validated on that data. Evolution is an iterative process of interaction, investment, and disinvestment. The temporal change of topological attributes for the network is also evaluated using measures of connectivity, density, heterogeneity, concentration, and connection patterns. The simulation model is validated using historical data from the Indiana interurban network. Statistical analyses suggest that the simulation model performs well in predicting the sequence of link abandonment in the interurban network as well as the temporal change of topological attributes. The simulation model is then applied on different idealized network structures. Typical connection patterns such as rings, webs, hub-and-spokes, and cul-de-sacs emerge in the networks; the spontaneous organization of network hierarchies, the temporal change of spacing between parallel links, and the rise-andfall of places in terms of their relative importance are also observed, providing further evidence for the self-organization property of surface transportation networks.
\end{abstract}

(c) 2008 Elsevier Ltd. All rights reserved.

\section{Introduction}

Scientific interest in the structure of complex networks have been aroused by the observation of a power-law distribution in a variety of so-called "scale-free" networks, such as the World Wide Web, metabolic networks, citation networks, and the network of human sexual contacts (Albert, Jeong, \& Barabasi, 1999; de Solla Price, 1965; Jeong, Gombor, Albert, Oltwai, \& Barabási, 2000; Liljeros, Edling, Amaral, Stanley, \& Aberg, 2001). As the physics community became interested in surface transportation networks, however, it was recognized that they exhibit topological attributes that differ from other classes of networks: Csányi and Szendröi (2004) demonstrated a clear dichotomy between large real-world networks which are small worlds with exponential neighborhood growth, and fractal networks with a power-law distribution. Typical examples of the latter are networks with strong geographical constraints, including power grids and surface transport networks; Gastner and Newman (2006), revealing that the structure of geographical networks are distinct from non-geographical ones, provided a connection between the two classes of networks in that they both can result from the same optimization model with one parameter varied. Specifically, Montis (2006) studied the interurban commuting network of the Sardinia region in Italy, and disclosed that the statistical properties of traffic structure exhibit complex features and non-trivial relations with the underlying

\footnotetext{
* Corresponding author. Tel.: +1 6126260024.

E-mail addresses: xiex0055@umn.edu (F.Xie), dlevinson@umn.edu (D. Levinson).

URLs: http://welelaw.umn.edu (F. Xie), http://nexus.umn.edu (D. Levinson).
}

topology; Jiang and Claramunt (2004) and Jiang (2005, 2007), after analyzing the street-street intersection topology (in which all named streets are represented as nodes, while street intersections as links) of urban street networks across North America and Europe, found that urban street networks exhibit a scale-free property characterized by a connectivity distribution with a power-law regime followed by a cutoff. The scale-free property with street topologies further suggests that "street networks or street topologies are selforganized" from an evolutionary perspective; Lämmer, Gehlsen, and Helbing (2006) analyzed urban road networks of the 20 largest cities in Germany and discovered scaling of several aspects of the networks, such as the number of nodes reachable within a travel time budget, which were only known for non-spatial networks. While the main efforts have been put to describe the topological dynamics of the networks in statistical physics (Barabasi \& Albert, 1999; Dorogovtsev \& Mendes, 2002), it remains unclear how surface transportation networks could spontaneously evolve into unique topological patterns as they grow and decline over time.

This question unavoidably requires an evolutionary view. The analysis and modeling of the evolution of transportation networks has been the subject of interest for more than half a century, and the literature has followed the following three main streams.

During the early days of the quantitative geography regional science economic geography movement, a few studies modeled the structural transformation of surface transportation networks. Kansky (1969) developed a quantitative predictive model of network structure and applied it to the Sicilian railroad. Taaffe, Morrill, and Gould (Taaffe, Morrill, \& Gould, 1963) proposed a four-stage model to describe the process of road network development in an 
undeveloped country. Garrison and Marble (1962) simulated the changing topology of the Northern Ireland railroad system between 1830 and 1930 using Monte Carlo methods, while Morrill(1965) reported parallel studies on the rail networks of central Sweden. These studies simply replicated the observation of network topologies using heuristics, while not taking into consideration inherent demographic and behavioral mechanisms that drive the evolution of transportation networks, largely due to limited data and computing abilities at that time. This stream of studies remained dormant for the following 30 years.

The attempts to extract or generate the optimal structure of networks represent another strand of evolutionary studies. Gastner and Newman (2006) presented an optimization model to minimize the cost of building and maintaining a network. Optimized network structures were able to replicate the qualitative features of the networks with or without spatial constraints, with one parameter in the cost function varied. Barthélemy and Flammini (2006) proposed a model of traffic networks via an optimization principle. The topology of the optimal network turns out to be a spanning tree and, by changing model parameters, different classes of trees are recovered. Schweitzer, Ebeling, Rose, and Weiss (1998) investigated the evolution of road networks during the optimization process by which a minimized travel detour is compromised with a minimized cost of constructing and maintaining roads. In the field of transportation planning, the prevalence of travel demand forecasting models since the 1970s made it possible to predict traffic flows on a transportation network in a more realistic way, thereby enabling the investigation into the optimal network structure that maximizes the efficiency of travel. In recent years, the travel demand model has been widely adopted to solve the network design problems (LeBlanc, 1975; Yang \& Bell, 1998), which derives the design of an optimum amount of transportation supply given the constraints of limited resources. Optimization studies have made significant contributions in predicting travelers' route choice behaviors at the demand level and optimal network structures at the supply level. These studies, however, assume changes to networks are made by a central authority subject to an explicit objective function, neglecting the continuous interplay between decision-makers, suppliers, and users with independent interests, which has served an essential role in shaping the structure of transportation networks from a long-term point of view. Moreover, little empirical evidence has been provided to show that the sequential deployment of transportation networks actually follows an optimal design.

In contrast to optimization, the concepts of agent-based interaction and self-organization have been introduced to interpret the evolution of various complex systems (Barabási, 2002; Newman, 2003). Agent-based simulation also found its application to interpret the formation of surface transportation networks based on simple individual travel behaviors. Lam and Pochy (1993) proposed an active-walker model (AWM) to describe the dynamics of a landscape, in which walkers as agents moving on a landscape change the landscape according to some rule and update the landscape at every time period. Helbing, Keltsch, and Molnr (1997) adopted the active walker model to simulate the emergence of trails in urban green spaces shaped by pedestrian motion. Starting from a homogenous ground, frequently used trails got reinforced since they are chosen by pedestrians more while rarely used trails withered. Consequently, the trails bundled and emerged into different patterns, which the authors claimed "reproduce many of the observed large-scale spatial features of trail systems." In recent years, limited efforts have been put to model the evolution of large-scale transportation networks employing agent-based simulation, bringing out some interesting findings regarding the emergent topological features of the networks studied. Yamins, Rasmussen, and Fogel (2003) presented a simulation of road growing dynamics on a land use lattice that generates global features as beltways and star patterns observed in urban transportation infrastructure, which however did not consider the dynamics of traffic flows. Yerra and Levinson (2005) and Levinson and Yerra (2006) demonstrated that a transportation network with a fixed structure can differentiate into a hierarchical structure from either a random or a uniform state, suggesting that the hierarchy of roads, rather than necessarily following an optimal design, is an emergent property of network and traffic dynamics.

To summarize the literature, network scientists have widely recognized that surface transportation networks, though different from non-spatial ones, also exhibit scale-free properties, suggesting surface transportation networks could be self-organized, although little evidence has been provided in this regard. Efforts to model the evolution of transportation networks have ranged from geographical studies that aim to replicate network geometries based on intuitive and heuristic rules, optimization studies that predict optimal network designs subject to an explicit objective function, to simulation studies that model network formation employing agent-based methods. These efforts, however, have been limited in three folds: first, with a few exceptions, many studies impose a top-down design of network structure, thereby neglecting the self-organization process that may drive the formation of transportation networks; second, the structural transformation of a network is not associated with a broader context that allows for the interactions between demand (travelers) and supply (infrastructure); third, little empirical evidence has been provided for the claim that existing models can replicate the topological change of transportation networks observed in reality.

The aim of this study is to fill these gaps. Differing from previous studies, our analysis implements a variable network topology in a spontaneous process of demand-supply interaction based on decentralized local optimal decisions. Another main contribution of this study is to validate the model against empirical facts extracted from historical observations and apply it to idealized networks, providing both empirical and simulation evidence for the self-organization property of surface transportation networks.

The rest of the paper takes the following form: we first introduce a simulation model that incorporates individual links as independently operating agents. While the weakest member in the network is shuttered, it enables a variable network topology forming from a bottom-up process. This is followed by a validation of the model using historical data from the Indiana interurban network. Then experiments are outlined applying the simulation model on idealized networks, and results presented. The conclusion summarizes our findings and indicates future directions.

\section{Simulation model}

The temporal development of a surface transportation network can be viewed as a degeneration process: starting from an underdeveloped area where all point-to-point paths can be used, those paths which are more valuable are reinforced while less used ones shrink and are finally abandoned. Taking road infrastructure as an example, while dirt trails and turnpikes built in the early stage of surface transportation disappeared on less used routes (such as those connecting villages to villages), those on valuable routes (such as those connecting towns to villages and to other towns) survived and were replaced by paved roads, some of which may be further upgraded into into arterial, highways or freeways. If our focus were on paved roads rather than the whole spectrum of road infrastructure, we would observe a network of paved roads that gets increasingly connected through time until they are replaced by infrastructure constructed with a newer technology. In this sense, the degeneration process represents the same "growth" process of transportation networks we have observed in reality. 
A simulation model is constructed in this study to replicate the evolutionary growth of transportation networks in a degeneration process. Alternatively, the model can be thought of as simulating a mature system where all links have been built, and some links are abandoned while others are improved. As a whole, the evolution of a network is represented as an iterative process over discrete time (simulation) periods and each period implements topological changes in a sequential process that includes five consecutive components: network loading, travel demand dynamics, investment, disinvestment, and topological measures, which will be explained in turn.

\subsection{Network loading}

The model treats the distribution of land use as exogenous inputs. The region is divided into land blocks or land use cells which hold the exogenous information of geographical location and demographics. A land use cell loads all the trips generated from this block onto the network at its nearest node. A land use cell will re-allocate its trips to the second nearest node if the node to which it is currently attached is removed. The distance from each land use cell to its nearest node is also re-calculated.

\subsection{Travel demand dynamics}

A traditional travel demand forecasting model includes four steps to predict the traffic flows on a given road network: trip generation, trip distribution, mode split, and traffic assignment (de Dios Ortuzar \& Willumsen, 2001). This study adopts the simplified travel demand model developed by Levinson and Yerra (2006), which considers only trips by a single abstract mode and assumes infinite link capacity in traffic assignment. The steps of trip generation, shortest path finding, trip distribution, and all-or-nothing traffic assignment are followed for each time period to predict the through traffic of each link.

According to the travel demand model, the total travel time $(T)$ that travelers spent for each time period can be calculated as follows:

$T=\sum_{(R, S)} q_{R S}\left(\frac{\overline{d_{R}}}{v_{0}}+\sum_{\mathrm{a}} \frac{l_{\mathrm{a}}}{v_{\mathrm{a}}} \delta_{a, R S}+\frac{\overline{d_{\mathrm{s}}}}{v_{0}}\right)$

where $q_{\mathrm{RS}}$ is the number of trips between any pair of nodes from the origin $R$ to the destination $\mathrm{S}$, estimated by the gravity model of trip distribution. ${ }^{1}$ The travel time of an average trip adds three parts: the first part calculates the average access time from the land use cells attached to $R$ to this node. The variable $\overline{d_{R}}$ is the average access distance from an attaching land use cell to node $R$, while $v_{0}$ is a specified minimal speed, which can be interpreted as the speed for accessing the closest network nodes from the land use layer. Similarly, the third part calculates the average egress time from destination $S$ to the land use cells attached to it. The second part sums the travel time spent on the links along the shortest path between $R$ and $\mathrm{S}$, where $\delta_{a, R S}^{i}$ is a dummy variable equal to 1 if link a belongs to this shortest path and 0 otherwise. The variable $l_{\mathrm{a}}$ indicates the length of link a and $v_{\mathrm{a}}$ indicates its average speed.

\subsection{Investment}

For each time period, each link agent invests in its speed level according to its revenues and costs in the preceding period. Suppose

\footnotetext{
${ }^{1}$ In analogy with physics, the gravity model illustrates the macroscopic relationship between homes and workplaces, in which the interaction between two locations declines with increasing time cost and monetary cost (user toll in this case) between them, but is positively associated with the amount of activity at each location (Isard, 1956).
}

link ab is an agent autonomously operating two parallel and opposite one-way links a and $b$ that connect two nodes. In each time period, the agent gathers revenues from the toll collected by both member links, computes the overall maintenance cost it spends on both links, and then decides to invest in its speed level.

Let $f_{\mathrm{a}}$ and $f_{\mathrm{b}}$, respectively represent the flows of link a and link $\mathrm{b}$ for iteration $i$. Earnings accruing to the link result from tolls that are collected by link agent ab as follows:

$E_{\mathrm{ab}}=\tau l_{\mathrm{ab}}\left(f_{\mathrm{a}}+f_{\mathrm{b}}\right)$

where $l_{\mathrm{ab}}$ is the length of each link (given that link a and link b have the same length) while $\tau$ represents the specified toll rate.

The cost to maintain links in their present usable conditions falls into two parts: the variable cost depends on link length, flow and speed, while the fixed cost is independent of flow and speed and only depends on link length. The overall maintenance cost spent by the agent operating links a and $\mathrm{b}$ for iteration $i$ is calculated by adding up the fixed and variable costs spent by both links as:

$C_{\text {fixed,ab }}=2\left(l_{\mathrm{ab}}\right)^{\alpha_{1}}$

$C_{\mathrm{var}, \mathrm{ab}}=\left(l_{\mathrm{ab}}\right)^{\alpha_{1}}\left[\left(f_{\mathrm{a}}\right)^{\alpha_{2}}+\left(f_{\mathrm{b}}\right)^{\alpha_{2}}\right]\left(v_{\mathrm{ab}}\right)^{\alpha_{3}}$

where $\alpha_{1}, \alpha_{2}$ and $\alpha_{3}$ represent specified length, flow, and speed powers in the cost equation, respectively.

It is assumed each link agent myopically decides to spend all available revenue at the end of each iteration without saving for the next time step. If the revenue (net of fixed costs) gathered from links $\mathrm{a}$ and $\mathrm{b}$ exceeds the maintenance cost spent for iteration $i$, remaining revenue will be invested to improve the speed level, that is, the running speed of links a and b. In contrast, if the revenue is insufficient to cover the cost, the running speed will drop (this speed degradation differs from the disinvestment process above which results in link abandonment). This myopic investment policy adopted by each link agent can be expressed as:

$v_{\mathrm{ab}}^{i+1}=v_{\mathrm{ab}}^{i}\left(\frac{\left(E_{\mathrm{ab}}^{i}-C_{\text {fixed }, \mathrm{ab}}^{i}\right)}{C_{\mathrm{var}, \mathrm{ab}}^{i}}\right)^{\beta}$

where $v_{\mathrm{ab}}^{i+1}$ and $v_{\mathrm{ab}}^{i}$ are respectively the speed levels of link agent ab for iteration $i+1$ and iteration $i$, while $\beta$ is a specified speed improvement coefficient.

\subsection{Disinvestment}

The disinvestment process eliminates the least used link(s) from the collection of existing links for each time period. In this case, the usage of a link is surrogated by the volume of its through traffic. The disinvestment criterion specifies how the weakest links are selected and ensures a minimal number of links to be eliminated for each time period. ${ }^{2}$ The idea of the "weakest link" heuristic arose from the concept of the "greedy algorithm" (Cormen, Leiserson, Rivest, \& Stein, 1990), in which locally optimum choice are made in a discrete optimization process at each stage with the hope of finding the global optimum. Similar to the greedy algorithm, the purpose of the "weakest link" assumption is not to be as realist as possible, but to capture the myopia of local optimal decisions made in a sequential process of transport development.

\footnotetext{
${ }^{2}$ For an asymmetric network, one and only one link agent that operates at the lowest flow level will be killed at a time among removable links. A removable link agent is one which, by removing both its member links, does not disconnect the network. For a symmetric network with symmetric demand, all the links on the symmetric positions operate at the same speed and flow level. Thus once a link agent is selected to be killed, all its symmetric counterparts will be selected automatically as well.
} 


\subsection{Measures of topological attributes}

At the end of each simulation period, a series of topological measures are computed to evaluate the collective structural features of the emergent network from four different dimensions, including the gamma index $(\gamma)$, the measure of network density $(D)$, the measure of entropy $(H)$, the Gini index $(G)$, and the measures of connection patterns.

The gamma index is one of a wide range of existing measures of connectivity that quantify the interconnection of nodes in a network (Harggett \& Chorley, 1969; Rodrigue, Comtois, \& Slack, 2006). The index compares the actual number of links with the maximum number of possible links in the network:

$\gamma=\frac{e}{3(v-2)}$

where $e$ is the number of edges (directional links) and $v$ is the number of vertices (nodes). Values for the gamma index range from 0 to 1 and a higher value represents a more connected network.

Network density measures the length of links per unit of surface. The higher it is, the more a network is developed. The density of a network $(D)$ is measured by the length of examined links $(L)$ divided by the area of the territory $(B)$.

$D=\frac{L}{B}$

The entropy of link speeds $(H)$ measures the heterogeneity (hierarchy) of roads in terms of their speeds, viewing a road network as a collection of individual roads. The concept of entropy was initially proposed to measure information uncertainty (Shannon, 1948). The speed entropy of a road network can be approximated by:

$H=-\sum_{k=1}^{\infty} p_{k} \log _{2}\left(p_{k}\right)$

where $p_{k}$ is the proportion of links of the $k$ th level with regard to the total number of existing links; speeds are organized into a histogram and links whose speeds fall into the range $k-1$ to $k$ are categorized into the $k$ th level.

Following Lämmer et al. (2006), the Gini index $G$ is adopted to reflect the concentration of traffic along links in a network. The importance of a links can be characterized by the number of vehicles or passenger that pass through it within some time interval. Accounting for the effect of heterogeneous link lengths, the actual use of a link is measured by the daily vehicle kilometer travel (VKT) or passenger kilometer travel (PKT) that occurs on a link. As the travel demand model allows the prediction of traffic volume on each link, the Gini index of VKT or PKT distribution on the network is approximated in a discrete form as follows:

$G=1-\sum_{k=1}^{e}\left(X_{k}-X_{k-1}\right)\left(Y_{k}+Y_{k+1}\right)$

where $X_{K}$ represents the cumulative portion of links for $k=0,1, \ldots, e$ while $Y_{K}$ represents the cumulative portion of total VKT or PKT. Links are sorted ascendingly according to VKT or PKT that occurs on individual links.

The measures of connection patterns including ringness ( $\left.\phi_{\text {ring }}\right)$, webness $\left(\phi_{\text {web }}\right)$, circuitness $\left(\phi_{\text {circuit }}\right)$, and treeness $\left(\phi_{\text {tree }}\right)$ are developed by Xie and Levinson (2007). An algorithm was developed to identify the pre-defined structural elements of ring, web, circuit, and branch in a network, and their relative significance can be evaluated as follows:

$\phi_{\text {ring }}=\frac{\sum_{i}\left(l_{i} \delta_{i}^{\text {ring }}\right)}{\sum_{i} l_{i}}$

where $l_{i}$ is the length of an individual link $i$; is equal to 1 when a link belongs to a ring. Similarly,

$\phi_{\mathrm{web}}=\frac{\sum_{i}\left(l_{i} \delta_{i}^{\mathrm{web}}\right)}{\sum_{i} l_{i}}$

Note that if a link is located on one and only one circuit, it belongs to a ring; if it is located on more than one circuit, it belongs to a web. If a link belongs to a web or ring, it is defined as a circuit link; otherwise it is defined as a branch link. Therefore,

$\phi_{\text {circuit }}=\phi_{\text {ring }}+\phi_{\text {web }}$
$\phi_{\text {tree }}=1-\phi_{\text {ring }}-\phi_{\text {web }}$

\section{Model validation}

Previous studies have revealed the similarity between topological patterns generated in their simulation models and those observed in reality. Visual similarity, however, is not sufficient for the validity of these models. This section validates our model by
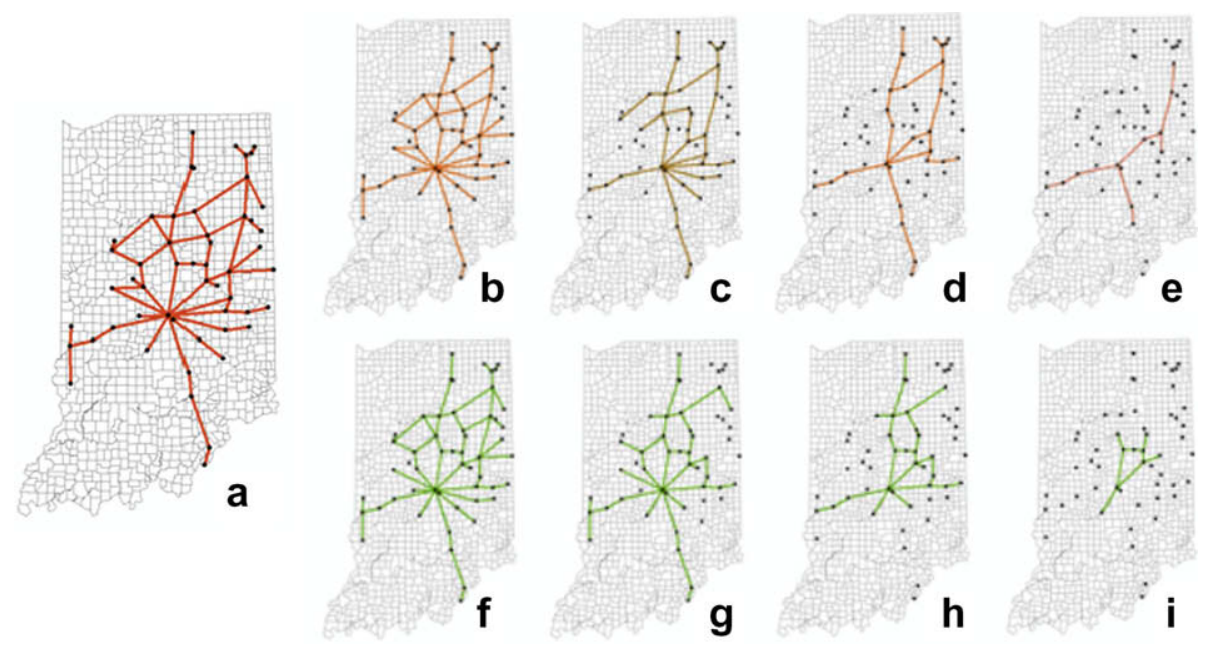

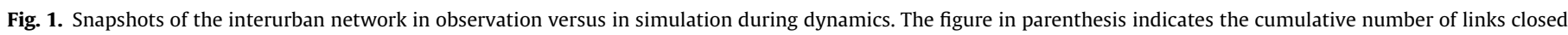

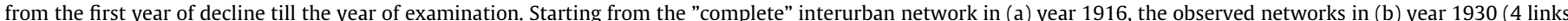

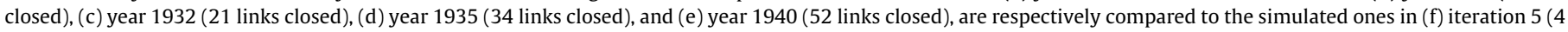
links closed), (g) iteration 22 (21 links closed), (h) iteration 35 (34 links closed), and (i) iteration 53 ( 52 links closed) with the same number of links remaining. 
extracting empirical data from a historic transportation network that actually experienced decline and comparing historical observations with simulation results based on statistical analysis.

Among those surface transport modes that have degenerated through time (canals, turnpikes, passenger rails, streetcars, interurbans, etc.), the interurbans in North America probably experienced the most dramatic change. Most interurban rails were built between 1901 and 1908, and by 1912 the interurban network had taken its final shape (15,500 miles or $25,000 \mathrm{~km}$ in the US). A marked decline set in about 1918, and within two decades the network was virtually annihilated. As the interurbans experienced such a short and relatively recent life cycle, their history is well documented and retrievable (Hilton \& Due, 1960).

Reaching a maximum of 1825 miles (2937 km), Indiana was second only to Ohio in the absolute size of its interurban network, and Indiana is the only state where a large-scale grid-like interurban network emerged. Therefore the Indiana network has been the subject of interest to researchers for a long time (Haley, 1936; Marlette, 1959). The network had its first line in 1887, started to decline from 1917, and completely disappeared in 1941. As shown in Fig. 1a, the interurban network of Indiana in 1916 represents the network in its full shape. ${ }^{3}$ As can be seen, the topological pattern of the network can be best described as a series of irregular wheels, with their spokes radiating from major cities such as Indianapolis and Fort Wayne. The decline phase of the network is taken as a case to validate our simulation model as follows.

Starting from the 1916 interurban network, a simplified travel demand model ${ }^{4}$ is adopted to estimate the traffic flows on individ-

\footnotetext{
${ }^{3}$ For the purpose of this study, the network of Indiana is separated from those other states basically along the state border. The portion in north Indiana is excluded because it was more connected to Chicago and the Michigan cities around Lake Michigan, than to the main body of the Indiana network. For simplicity, detour lines shorter than 2 miles $(3.2 \mathrm{~km})$ are neglected and the locations of the places as the terminals of these lines are adjusted as if they were located on the main line, including Milton, Richmond, Gas City, and Riverside Park. Consequently, a network of total 53 places and 62 interurban rail segments (124 one-way links) is identified.

4 The travel demand model takes population data by county as exogenous input. The state of Indiana consists of 92 counties, and the historical population records of each county are available every decade from 1890 to 1990 (Forstall, 1990). Population of each county for each year was estimated by interpolation. Assuming the population of a county is allocated to the nearest station (city), measured by the linear distance from the centroid of this county to the station, 10 out of 53 stations will be assigned no passengers, which is obviously unrealistic. To resolve this issue, the census tract is instead used as the geographical unit to hold population information. The population of a county is assumed to be evenly distributed. With no historical population data by census tracts available in digital form, the population of a county is assumed to be evenly distributed and allocated to tracts within its boundary in proportion to their areas. Trips generated by each tract are then allocated to the nearest station. Considering people have to access the stations on foot or by horse or carriage at that time (auto ownership was not yet widespread, and auto drivers would be less likely to use an interurban), it is also assumed that only people living in the tracts within 20 miles $(32 \mathrm{~km})$ stations would consider taking interurban rail. An estimated $94.4 \%$ of the revenues of electric lines in 1902 came from passenger traffic (Hilton \& Due, 1960). Thus this study considers only passenger traffic on the network, and estimates trip generation from a station as a linear function of its assigned population. It is assumed that each person will generate one trip per annum by interurban regardless of other modes. As no historical evidence shows that the interurban had a notable speed change, the interurban speed is assumed to be fixed over time and uniform over space. Specifically, it is assumed that interurban lines have a uniform generalized speed of $15 \mathrm{mi} / \mathrm{h}(24 \mathrm{~km} / \mathrm{h})$ (taking into consideration a uniform fare per mile) while centroid connectors have a speed of $5 \mathrm{mi} / \mathrm{h}(8 \mathrm{~km} / \mathrm{h})$. A doubly constrained trip distribution model is adopted and the decay factor is set as 0.01 as it is in the simulation experiments. A doubly constrained trip distribution model is adopted and the decay factor is set as 0.01 as it is in the simulation experiments, and an all-ornothing assignment is performed which assigns all the trips between an origin station and a destination station on the shortest path between both stations. Note that the predicted traffic flows on individual links need not be calibrated against the actual traffic level because the "weakest link" heuristic is concerned only with the relative flow level on links. Thus the parameters in trip generation, although arbitrarily specified, will not affect the predicted course of link abandonment. The sensitivity of model results on the decay factor in the trip distribution model, on the other hand, will be examined.
}

ual links, and the link operated on the lowest flow level is removed for each time period based on the "weakest link" heuristic until the whole network disappears (i.e. in this case no stopping rule is imposed). A Spearman's rank-order correlation test (Higgins, 2003) is then taken to correlate the predicted sequence of link closure in simulation with the actual sequence of link closure (in which links are ranked according to the actual years of closure extracted from historic records). Spearman's rank-order correlation test assesses how well an arbitrary monotonic function describes the relationship between two variables, without making any assumptions about the frequency distribution of the variables.

According to the rank-order correlation test, the correlation coefficient is equal to 0.287 . The $z$-test of correlation significance scores -2.245 with the $p$-value equal to 0.024 , suggesting the two sequences are positively correlated at a $95 \%$ significance level. The correlation test implies that although based on a simple heuristic that the links with the least traffic will be closed first, the "weakest link" heuristic predicts well the sequence of link closure during the decline phase of the Indiana interurban network. Additional runs with different decay rates in the travel demand models show that the correlation is robust over the change of the decay factor and is even more prominent with a smaller decay rate. ${ }^{5}$

Fig. $1 \mathrm{~b}-\mathrm{i}$ present the snapshots of the interurban network during the decline process in simulation versus in observation. To better illustrate, the topological change of the network is depicted in Fig. 2 as the fluctuations of proposed topological measures both from simulation and from observation (the measure of circuitness is not included but treeness provides a complement measure). As can be seen in Fig. 2c, the measures of ringness in simulation indicate the emergence and collapse of a ring in the network, which is exactly what we observed in reality, although it is several iterations lagged as compared to reality. The series of each topological measure in simulation are compared to their counterparts in observation using Two-sample Wilcoxon rank-sum (Mann-Whitney) test, which is a non-parametric significance test assessing if two independent samples come from the same population (Higgins, 2003). Viewing the measures over time periods as time-series data, this test essentially assesses if two series of data fluctuate over time following the same trend. The statistical results for each topological measure are also presented. Provided that the null hypothesis of the rank-sum test is that the two samples are drawn from a single population, none of the presented $z$-values provide sufficient evidence to reject the null hypothesis. Thus we can conclude that the computed attributes in simulation approximate the actual topological attributes of the network over time.

It is important to point out that, although empirical evidence from Indiana Interurbans lends credibility to our model, it does not necessarily mean this model can be universally applied to other transportation networks with different scales, of different modes, or in different regions without re-calibration or modifications. After all, given the complexity transport development involves, the purpose

\footnotetext{
5 The decay factor in the trip distribution model indicates the rate of decline of the interaction between places across the interurban network. To test the sensitivity of the statistical results on the decay factor, model validation was re-executed with two different values of 0.005 and 0.02 , and the Spearman correlation coefficient was recalculated. With a lower rate of decline of the interaction (0.005), the Spearman correlation test results in a more significant correlation (the correlation coefficient equals 0.429 with a $p$-value of 0.0006 ), suggesting that under a higher degree of interaction, the "weakest link" heuristics performs even better in predicting the sequence of link abandonment. With a higher decline rate of 0.02 , on the other hand, the Spearman correlation coefficient, though still with the positive sign, is much smaller and statistically not significant (the correlation coefficient equals 0.125 with a $p$-value of 0.289). This could be explained by the fact that as the decay factor increases, people become reluctant to travel farther. Provided that the interurban lines attract mainly intercity travel, the travel demand on the network may be underestimated, especially on strategic routes that connect big cities. This accordingly undermined the predictive performance of the "weakest link" heuristics.
} 

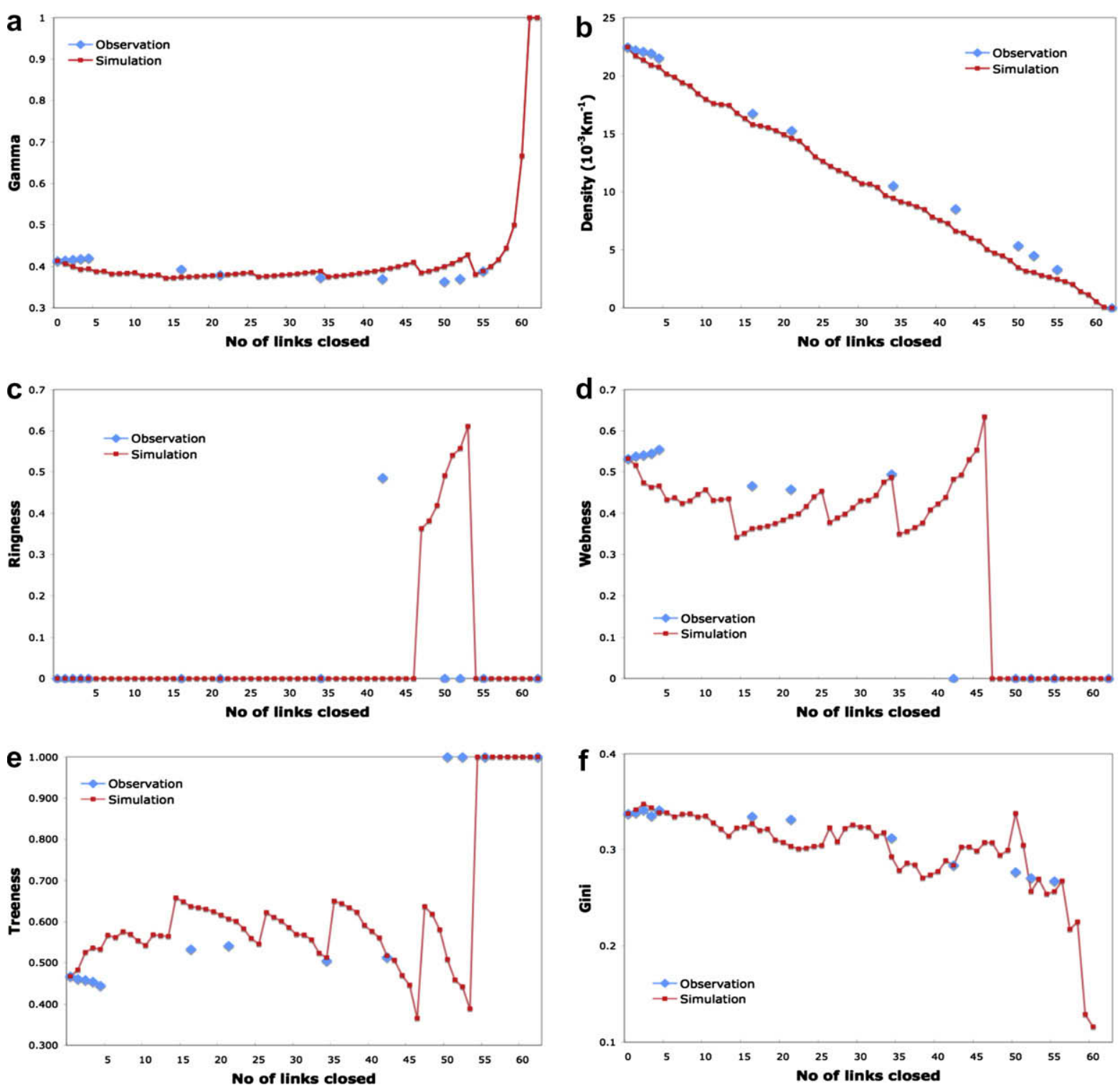

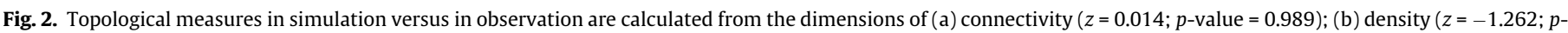

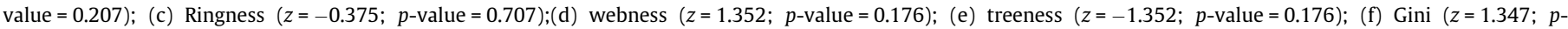
value $=0.178$; ; the results of the Mann-Whitney test are also presented comparing the series of measures in simulation versus that in observation.

of our model is not to be realist as possible, but to capture the selforganization property of transportation networks in an evolutionary process based on heuristic assumptions.

\section{Simulation experiments}

As the simulation model is validated on the Indiana interurban network, it is applied on idealized network structures with different initial conditions to explore (1) the temporal change of topological attributes from different network structures (2) typical topological properties that emerge during the autonomous evolution of networks, and (3) the sensitivity of emergent patterns to initial network conditions and to the parameters of the simulation model. A different stopping rule is also used. To ensure the connectivity of the network, an existing node must connect to at least another existing node, and neither isolated nodes nor sub-networks are allowed during the disinvestment process. In the idealized ultra-connected networks developed for this study, a secondary node will be removed if all links connected to it are removed while a primary node is not removable and it must be connected by at least one link.

\subsection{Idealized network structures}

Most cities have rather complex geometries of surface transport infrastructure. But from most origins in a city, one can travel locally in only four directions on its surface transportation network. In this sense, a surface transportation network has properties similar to a rectangular grid, in which travelers can only make a turn of $90^{\circ}$ or a multiple of $90^{\circ}$ from each node. The more directions which one can travel from any point, the shorter will be the average travel distance between points; at the same time the network will be more redundantly connected (Newell, 1980).

Aside from a grid network that simulates an actual urban road network, two ultra-connected networks are also tested, respectively referred to as the complete network and the hexagon network, which represent undeveloped networks where more directions are available moving from point-to-point.

A network developed by directly connecting every pair of two nodes among an original node set, which come from the intersection nodes of a grid network, is referred to as a complete network in this study. When links intersect, a new secondary node is created, 
and the longer link is replaced by shorter links that ultimately connect the same original nodes. A complete network does not necessarily directly connect each secondary node to an original node or another secondary node. Where links overlap, the longer link is eliminated. Note that theoretically the turning directions included in a complete network range from $0^{\circ}$ to $360^{\circ}$, depending on the size of the original grid network.

A hexagon network is also developed based on a grid network, in which the included angle of two intersecting links can be $30^{\circ}$ or its multiples. Note that in the hexagon network, the primary nodes with 12 links connected are scattered on a lattice of equilateral triangles, and the secondary nodes connected to a primary node form a hexagon. This type of network has the same topology as the hexagonal landscape developed according to the transportation principle of central place theory (Christaller, 1933; King, 1985), where smaller places are always located on the major transportation routes between the nearest larger places.

Since all these networks are based on a square grid, their size can be indicated by the number of nodes along each side of the original square grid. For example, a $3 \times 3$ complete network is a network developed on a $3 \times 3$ grid. With the extent of a grid network fixed, its size also determines the average length of links and the spacing of parallel roads in the network.

These idealized networks are developed to represent transportation networks with geographical constraints, which as disclosed by Csányi and Szendröi (2004) are subject to the following scaling law:

$N_{\mathrm{S}}(r) \sim r^{d}$

where $N_{\mathrm{S}}(r)$ denotes the size of neighborhood of node $\mathrm{S}$ within radius $r$, i.e., the number of nodes which can be reached from $S$ in at most $r$ steps. The scaling exponent is defined by Gastner and Newman (2006) as the effective dimension of a network. In an infinite network it is calculated as:
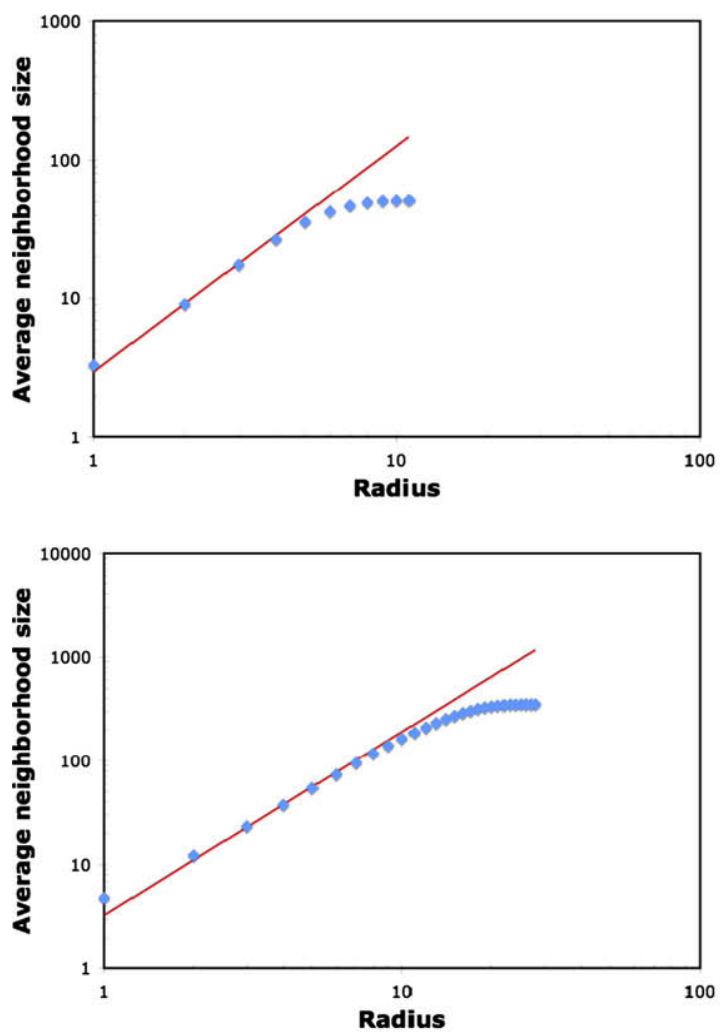

$d=\lim _{r \rightarrow \infty} \frac{\log N_{\mathrm{S}}(r)}{\log r}$

As transportation networks are finite, Lämmer et al. (2006) approximated the calculation by computing the average neighborhood size from a node in the network, plotting it over radius in double logarithmic scale. The slope of the curve at its inflection point gives the lower bound for an estimate of the dimension.

Fig. 3 displays the estimated dimension of each idealized network, as well as that of the Indiana Interurban network in 1916 for comparison. As can be seen, the idealized networks demonstrate scaling properties that are similar to the Interurban network but distinct from scale-free networks, that is, the estimated dimensions for all the networks are strictly between 1 and 2, representing networks on the two-dimension surface of the Earth with limited sizes.

\subsection{Simulation experiments and parameters}

The model is run on different initial specifications in a series of six experiments listed in Table 1 . The simulator of ultra-connected network disinvestment (SOUND) is developed to implement the simulation model and visualize the results. Specifically, links are classified into five levels according to their speed values $(0.01-5$, $5-10,10-15,15-20,20+)$, with different levels of links displayed with different colors and thickness.

A square 2500-cell land use layer is adopted for all the experiments while three different network structures are tested: the $10 \times 10$ grid network, the $4 \times 4$ complete network, and the $15 \times 15$ hexagon network. For simplicity, a uniform distribution is specified for land uses under which each land use cell generates and attracts 10 trips at the beginning of each time period. The initial speeds of links are also pre-specified as exogenous inputs and they determine the symmetry of a network. A network with a symmetric
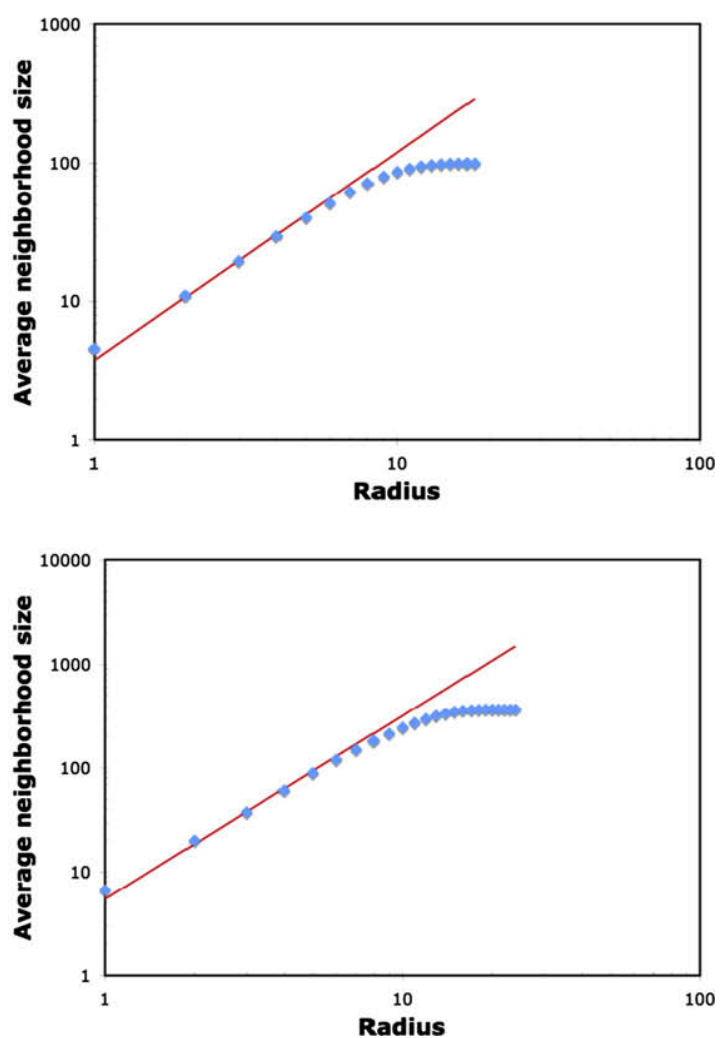

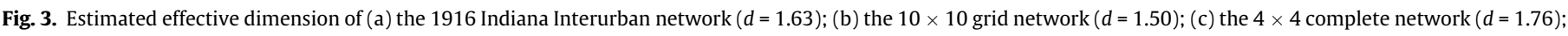
and $(d)$ the $15 \times 15$ hexagonal network $(d=1.77)$. 
Table 1

Specifications of experiments

\begin{tabular}{lllll}
\hline \multirow{2}{*}{ No. } & \multicolumn{2}{l}{ Initial conditions $^{\mathrm{a}}$} & & \multirow{2}{*}{ Iterations } \\
\cline { 2 - 4 } & Network & Link speeds & Land use & \\
\hline 1 & $10 \times 10$ grid & Random & Uniform & 200 \\
2 & $10 \times 10$ grid & Uniform & & 50 \\
3 & $4 \times 4$ complete & Random & & 800 \\
4 & $4 \times 4$ complete & Uniform & & 120 \\
5 & $15 \times 15$ hexagon & Random & & 1000 \\
6 & $15 \times 15$ hexagon & Uniform & & 300 \\
\hline
\end{tabular}

a Random speeds are initial speeds specified for each link which are randomly distributed from 1 to 10 ; uniform speeds are initial speeds specified for each link which are equal to 5; uniform land uses are specified initial land uses which generate 10 trips from and attract 10 trips to each cell.

Table 2

Model parameters and their specified values

\begin{tabular}{lll}
\hline Parameters & Description & Value \\
\hline$v_{0}$ & Walking speed in Eq. (1) & 0.01 \\
$\tau$ & Toll rate in Eq. (2) & 1 \\
$a 1$ & Length power in Eq. (3) & 1 \\
$a 2$ & Flow power in Eqs. (3) and (4) & 0.75 \\
$a 3$ & Speed power in Eqs. (3) and (4) & 0.75 \\
$\beta$ & Speed improvement coefficient in Eq. (5) & 1 \\
\hline
\end{tabular}

base structure, uniform land uses, and uniform link speeds evolves symmetrically in its topology, and thus it is called a symmetric network. Experiments 2, 4, and 6 were implemented on symmetric networks with uniform initial speeds and a symmetrical algorithm is included to ensure that the network evolved symmetrically; on the other hand, a network with random initial link speeds evolves asymmetrically and it is called an asymmetric network. Experiments 1,3 , and 5 were implemented on asymmetric networks with random initial speeds. Experiment 3 was repeated three times with different sets of random initial speeds, labeled as 3a, 3b, and 3c, respectively. Before the disinvestment process is started, the network growth model presented by Levinson and Yerra (2006) is applied with the network topology fixed. Since all the experiments reached a stable equilibrium in the network growth model before the 20th iteration, the disinvestment process was started at the 20th iteration and iterated until the minimally connected network was derived. After the disinvestment process was terminated, the network growth process continued until it became stable again. Note also that the minimal number of links to be eliminated depends on the number of axes in a symmetric network. For example, in a complete network with 3 axes, up to 8 link agents, i.e., 16 oneway links will be removed at a time.

Table 2 lists model parameters and their values for these experiments. The values are specified based on our best knowledge of economies of scale in transportation economics, and the sensitivity to these parameters will be discussed later.

\subsection{Experimental results}

To illustrate the dynamics of network topologies in simulation, the snapshots of test networks in Experiments 1-6 are respectively displayed, including the initial network, an interim network, and a

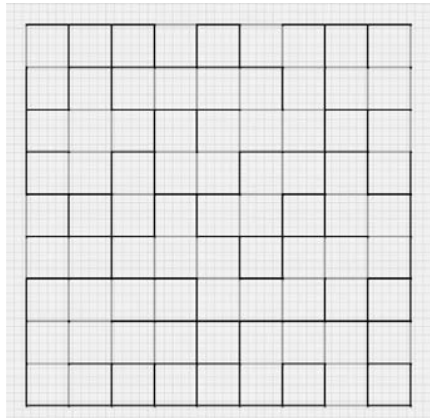

Iteration 0

$\gamma=0.61 \quad D=0.87 \quad H=3.12 \quad G=0.44$

$\phi_{\text {ring }}=0.0 \quad \phi_{\text {web }}=0.0 \quad \phi_{\text {tree }}=0.0$

b

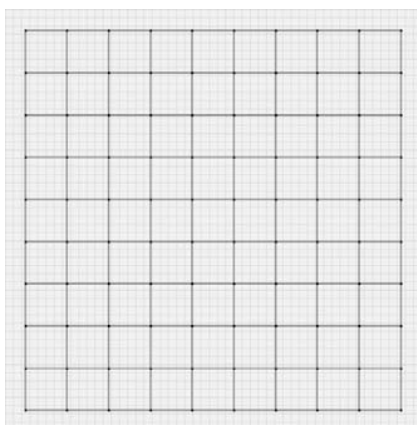

Iteration 0

$$
\gamma=0.61 \quad D=0.87 \quad H=0.0 \quad G=0.17
$$$$
\phi_{\text {ring }}=0.0 \quad \phi_{\text {web }}=0.0 \quad \phi_{\text {tree }}=0.0
$$

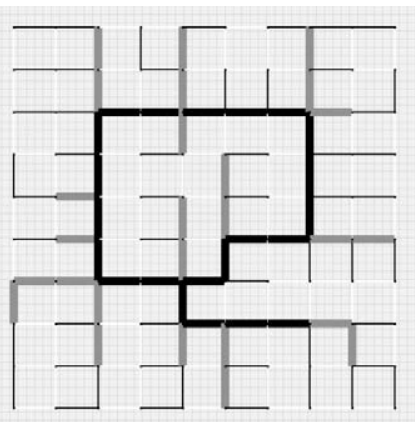

Iteration 100

$\gamma=0.340 \quad D=0.483 \quad H=3.15 \quad G=0.56$

$\phi_{\text {ring }}=0.37 \quad \phi_{\text {web }}=0.0 \quad \phi_{\text {tree }}=0.63$

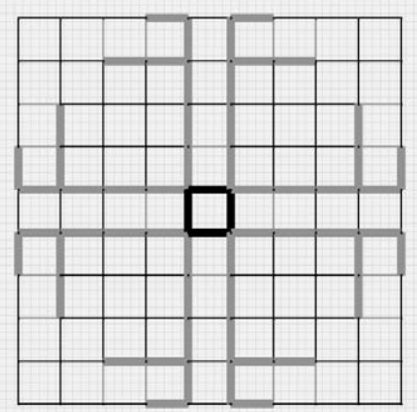

Iteration 20

$$
\begin{array}{cccc}
\gamma=0.61 & D=0.87 \quad H=3.31 \quad G=0.46 \\
\phi_{\text {ring }}=0.07 \quad \phi_{\text {web }}=0.0 \quad \phi_{\text {tree }}=0.93
\end{array}
$$
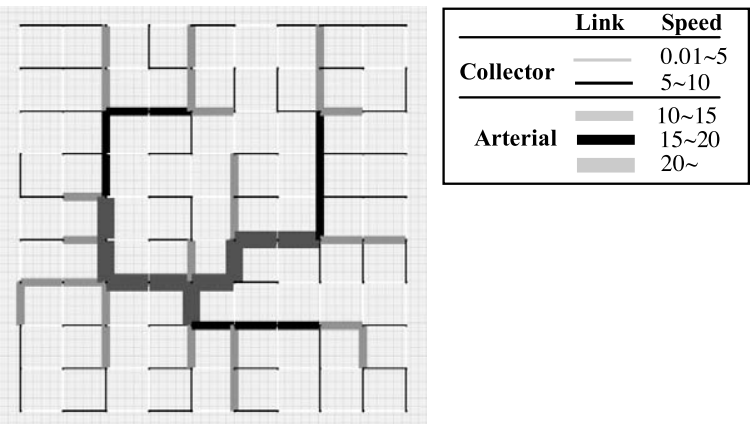

Iteration 200

$\gamma=0.337 \quad D=0.478 \quad H=3.21 \quad G=0.56$

$\phi_{\text {ring }}=0.0 \quad \phi_{\text {web }}=0.0 \quad \phi_{\text {tree }}=1.0$

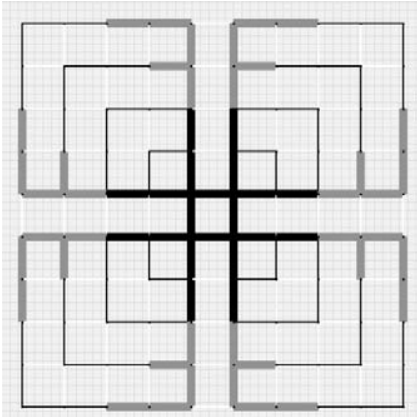

Iteration 50

$$
\gamma=0.39 \quad D=0.56 \quad H=3.20 \quad G=0.48
$$$$
\phi_{\text {ring }}=0.07 \quad \phi_{\text {web }}=0.0 \quad \phi_{\text {tree }}=0.93
$$

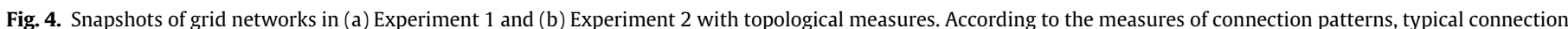

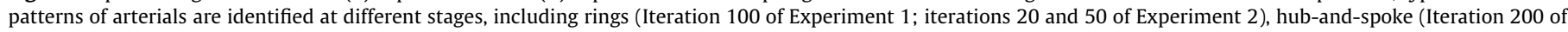
Experiment 1), and cul-de-sacs (Iterations 150 and 200 of Experiment 1). The legend bar applies to idealized networks in other figures as well. 
the final minimally connected network. Proposed topological measures of these networks are also presented. To reduce the running time, the measures were computed every five iterations. As most of the networks have rather complicated topologies, the links are classified into arterials and collectors according to their absolute speeds (links with their speeds above 10 are categorized into arterials) and the measures of connection patterns are computed only for the arterials whose topological patterns are of greater importance in a network.

\subsubsection{Experiments on grid networks}

The snapshots of an evolving grid network in asymmetric (Experiment 1) and symmetric (Experiment 2) scenarios are displayed in Fig. 4. As can be seen, both scenarios generate hierarchical network topologies over time, but an asymmetric network evolves into a tree-like structure with cul-de-sacs, while a symmetric network evolves into four symmetric sectors connected by a ring in the center.

\subsubsection{Experiments on complete networks}

The snapshots of Experiments 3a and 4 are displayed in Fig. 5, starting from the $4 \times 4$ complete network with random and uniform speeds, respectively. Fig. 6 displays different minimally connected networks derived from Experiment $3 a-c$ for comparison.

The topological change of the networks can be further corroborated by the fluctuations of topological measures over iterations. Taking Experiment 3a as an example, Fig. 7 plots the measures of network topology from different dimensions. In Fig. 7a, the disinvestment process is characterized by a continuous decrease of connectivity (the gamma index), as the weakest links are repeatedly removed from the network. Not surprisingly, the fluctuation of network density displays a similar downward-sloping pattern. The dynamics of speed entropy, as shown in Fig. 7b, displays more fluctuations: the initial disordered status with a random distribution of link speeds corresponds to a high initial value of speed entropy (3.18). As some links at lower levels are abandoned while others become faster and enter higher levels, the entropy slopes downwards until about the 400th iteration, when most links serve as collectors operated at a speed below 10 . Then the entropy gradually increases from about 2.10 to 2.60 because more and more links are improved to arterials. When the network shrinks close to the minimal size, a substantial jump of the entropy is observed at the 780th iteration. As can be seen, the entropy measure reflects how the heterogeneity of the structure changes over time as autonomous links develop into different hierarchies. Fig. 7c displays the change of the Gini index, which indicates the concentration of traffic on the network. The curve slopes down as links with lowest volumes are removed and traffic becomes less concentrated across remaining links, while a substantial increase is observed at the 780th iteration. Fig. 7d depicts the change of the connection patterns of arterials in terms of their relative significance since the first arterial appears at the 170th iteration. As more and more links improve themselves into arterials, individual arterial links that appear at scattered locations on different routes eventually merge into a contiguous network. The arterial network becomes more and more connected in the beginning, reflected by a decrease of treeness and an increase of a

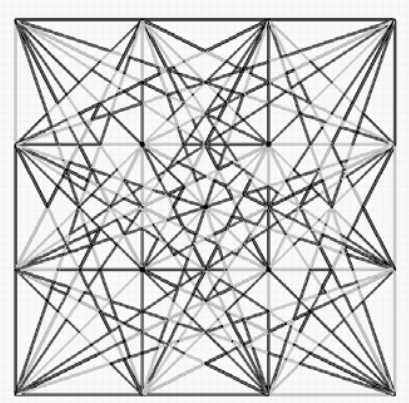

Iteration 0

$\gamma=0.83 \quad D=2.46 \quad H=3.18 \quad G=0.47$

$\phi_{\text {ring }}=0.0 \quad \phi_{\text {web }}=0.0 \quad \phi_{\text {tree }}=0.0$

b

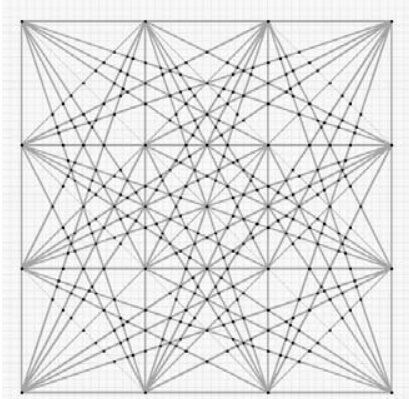

Iteration 0

$\gamma=0.83 \quad D=2.46 \quad H=0.0 \quad G=0.37$

$\phi_{\text {ring }}=0.0 \quad \phi_{\text {web }}=0.0 \quad \phi_{\text {tree }}=0.0$

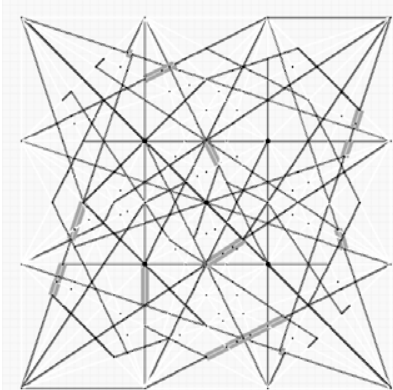

Iteration 400

$\gamma=0.52 \quad D=1.21 \quad H=2.17 \quad G=0.35$

$\phi_{\text {ring }}=0.0 \quad \phi_{\text {web }}=0.0 \quad \phi_{\text {tree }}=1.0$

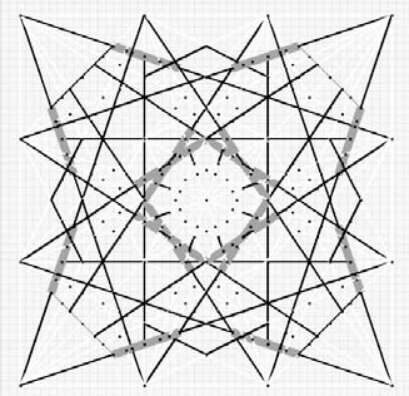

Iteration 80

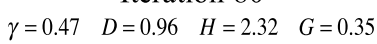

$\phi_{\text {ring }}=0.31 \quad \phi_{\text {web }}=0.0 \quad \phi_{\text {tree }}=0.69$

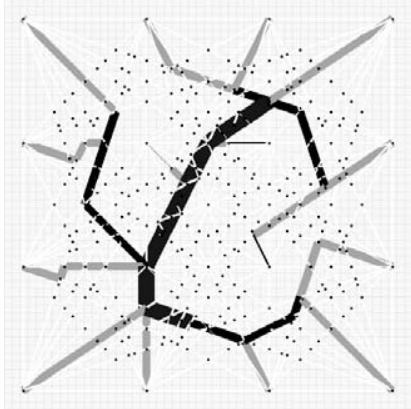

Iteration 800

$\gamma=0.34 \quad D=0.28 \quad H=3.52 \quad G=0.40$

$\phi_{\text {ring }}=0.0 \quad \phi_{\text {web }}=0.0 \quad \phi_{\text {tree }}=1.0$

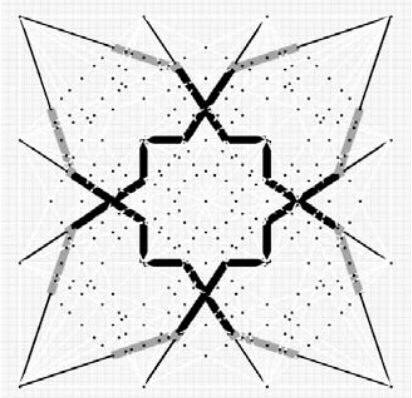

Iteration 120

$\gamma=0.39 \quad D=0.56 \quad H=3.31 \quad G=0.40$

$\phi_{\text {ring }}=0.35 \quad \phi_{\text {web }}=0.0 \quad \phi_{\text {tree }}=0.65$

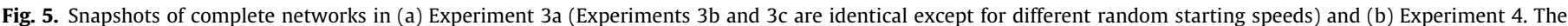

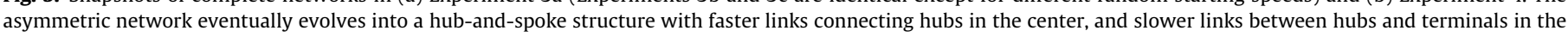

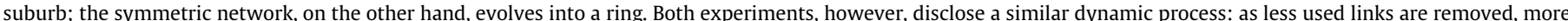

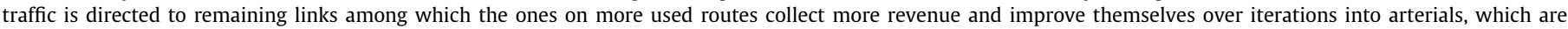
initially scattered in the network, and then emerge into a contiguous arterial network. 


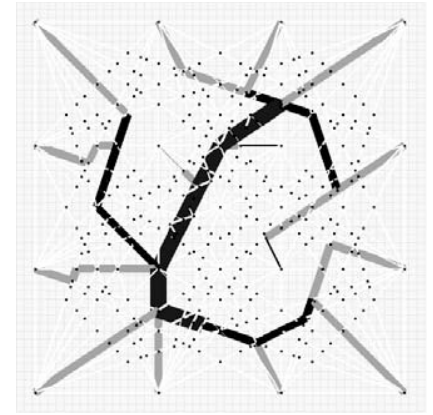

Experiment 3a

$\gamma=0.34 \quad D=0.28 \quad H=3.52 \quad G=0.40$

$\phi_{\text {ring }}=0.0 \quad \phi_{\text {web }}=0.0 \quad \phi_{\text {tree }}=1.0$

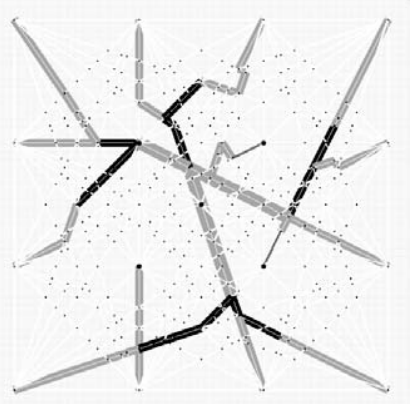

Experiment $3 \mathrm{~b}$

$\gamma=0.34 \quad D=0.27 \quad H=3.45 \quad G=0.37$

$\phi_{\text {ring }}=0.0 \quad \phi_{\text {web }}=0.0 \quad \phi_{\text {tree }}=1.0$

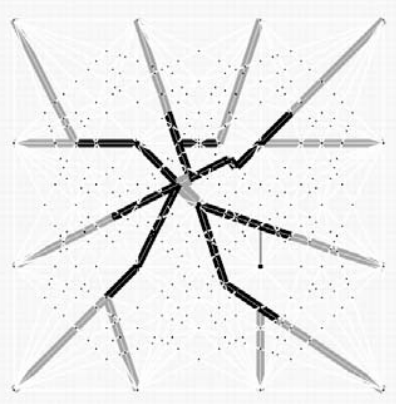

Experiment 3c

$\gamma=0.34 \quad D=0.26 \quad H=3.14 \quad G=0.33$

$\phi_{\text {ring }}=0.0 \quad \phi_{\text {web }}=0.0 \quad \phi_{\text {tree }}=1.0$

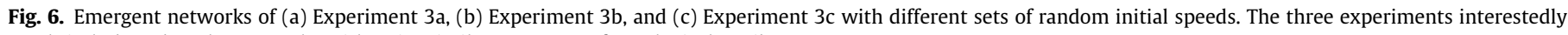
result in hub-and-spoke networks with quite similar measures of topological attributes.
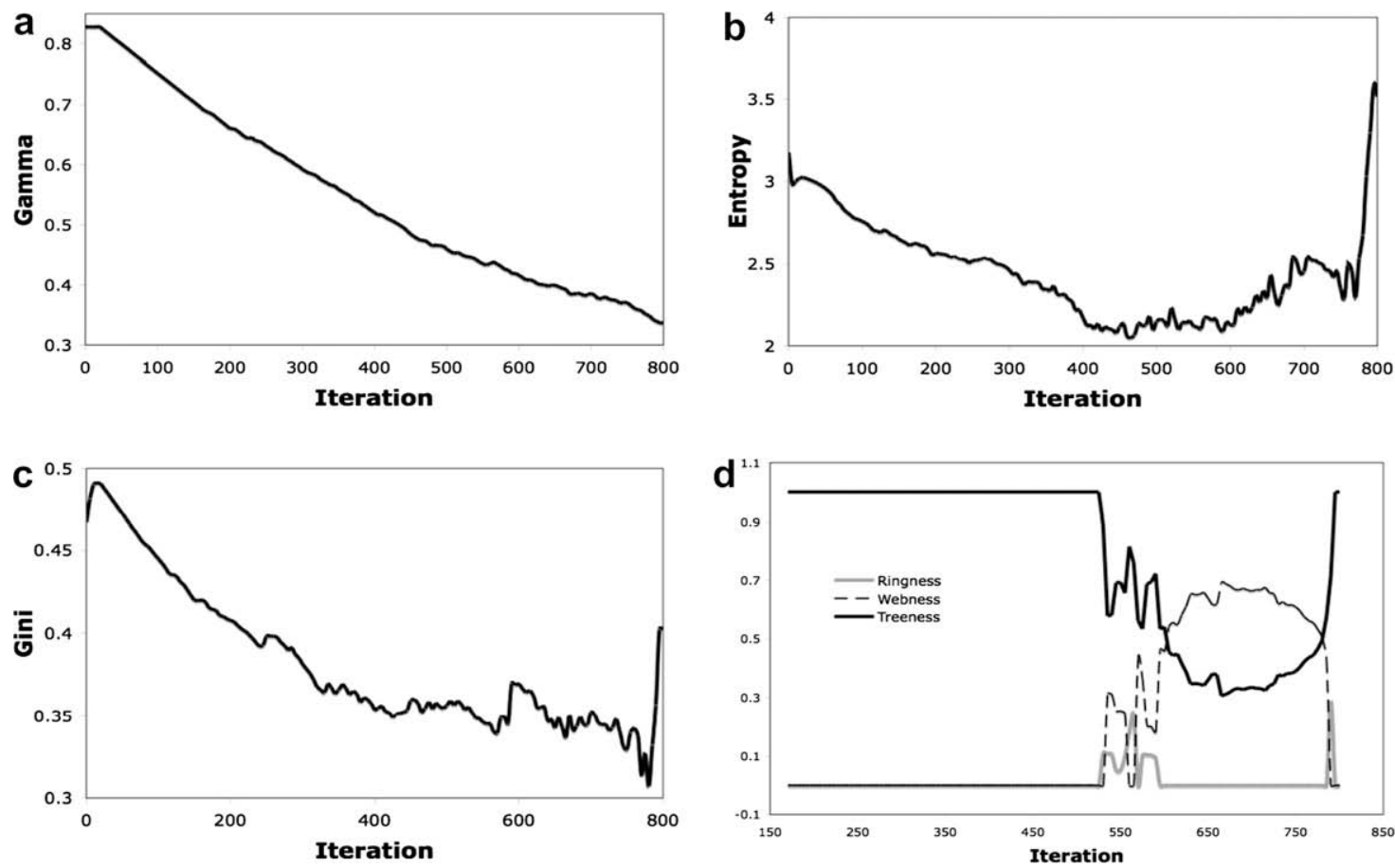

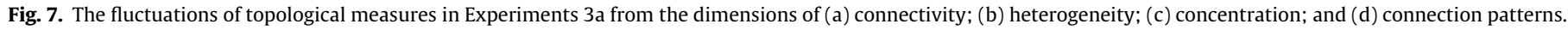

webness, since the first ring appears in the 530th iteration and the first web in the 535th iteration. When all the remaining links become arterials and weaker arterial links have to be removed from the network, however, the arterial network shrinks and eventually evolve into a hub-and-spoke structure. In a reverse process, the treeness increases while the webness and ringness decrease. It is worthy noting that the collapse of the last ring (when measure of ringness drops to null) occurs also at the 780th iteration, when speed heterogeneity (entropy) and traffic concentration (Gini) substantially increase. This could be explained by the fact that the significant transformation of network structure leads to the redirection of a large volume of traffic on the network when a few links remain.

\subsubsection{Experiments on hexagon networks}

The snapshots of Experiments 5 and 6 are displayed in Fig. 8. Note that the original hexagon network is designed with nodes evenly distributed on a homogenous landscape. Initially, different levels of nodes (differentiated by number of connections) have different scopes of "market", within which the cells are allocated to the most dominant place. Without considering the nodes on the border of the network, the primary nodes (centers of hexagons) are allocated more cells. Thus they are expected to represent more important places which serve more traffic. As the network evolves, however, the function and relative importance of places changed. As arterials connected into spokes or beltways, minor places along these links are reinforced, eventually serving a majority of though traffic. This finding suggests that instead of assuming a static landscape as in the central place theory, the potential impact from an evolving transportation network on the land use pattern needs to be incorporated for a more realistic simulation of network dynamics.

Another interesting finding is the temporal change of link spacing. There exist 12 directions for traffic from each hexagon center in the initial hexagon network. Along 6 directions, parallel links have smaller spacing. When the network becomes less dense, as we can 
a

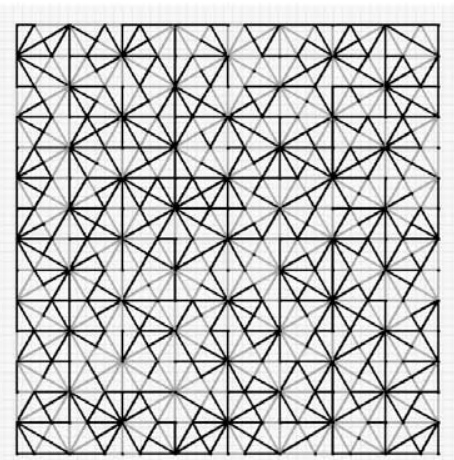

Iteration 0

$\gamma=0.95 \quad D=2.94 \quad H=3.17 \quad G=0.44$
$\phi_{\text {ring }}=0.0 \quad \phi_{\text {web }}=0.0 \quad \phi_{\text {tree }}=0.0$

b

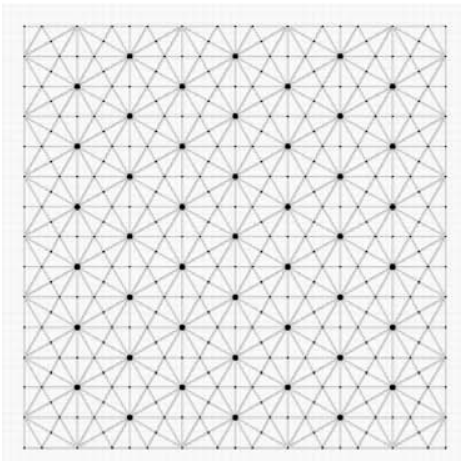

Iteration 0

$$
\begin{gathered}
\gamma=0.95 \quad D=2.94 \quad H=0.0 \quad G=0.35 \\
\phi_{\text {ring }}=0.0 \quad \phi_{\text {web }}=0.0 \quad \phi_{\text {tree }}=0.0
\end{gathered}
$$

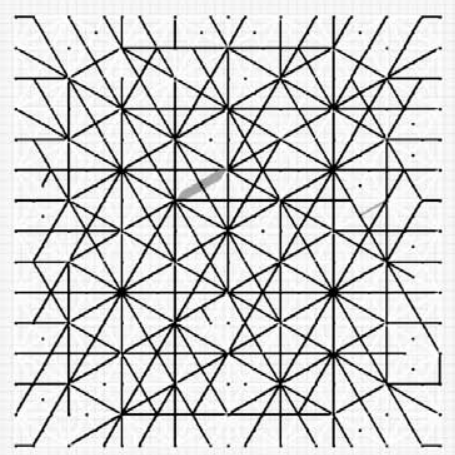

Iteration 500

$\gamma=0.55 \quad D=1.57 \quad H=2.24 \quad G=0.30$

$\phi_{\text {ring }}=0.0 \quad \phi_{\text {web }}=0.0 \quad \phi_{\text {tree }}=1.0$

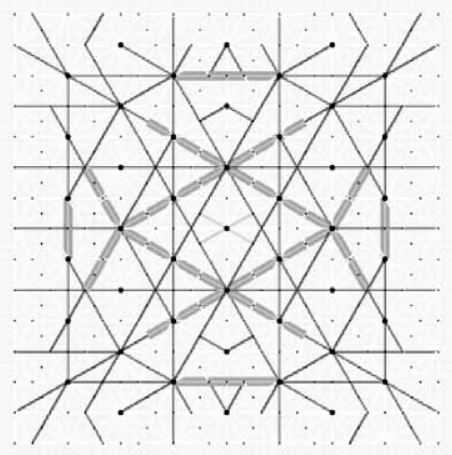

Iteration 200

$$
\gamma=0.46 \quad D=0.96 \quad H=2.23 \quad G=0.25
$$$$
\phi_{\text {ring }}=0.33 \quad \phi_{\text {web }}=0.0 \quad \phi_{\text {tree }}=0.67
$$

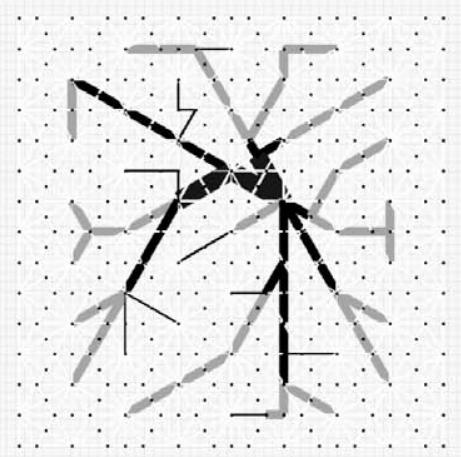

Iteration 1000

$\gamma=0.34 \quad D=0.31 \quad H=3.62 \quad G=0.42$

$\phi_{\text {ring }}=0.0 \quad \phi_{\text {web }}=0.0 \quad \phi_{\text {tree }}=1.0$

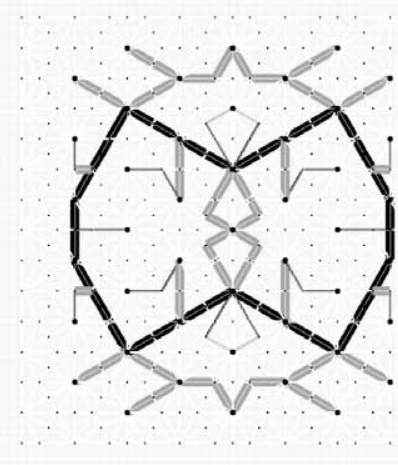

Iteration 300

$\gamma=0.36 \quad D=0.36 \quad H=3.17 \quad G=0.37$

$\phi_{\text {ring }}=0.0 \quad \phi_{\text {web }}=0.70 \quad \phi_{\text {tree }}=0.30$

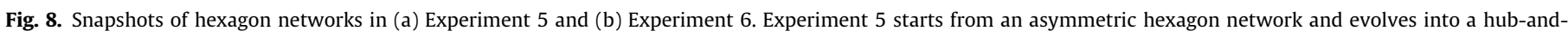

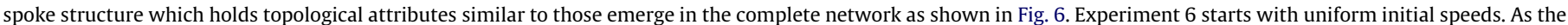
network grows, scattered arterials emerge and connect into a ring at the 200th iteration, and finally expand into a mesh-like network.

observe in Fig. 7a (Iteration 500) and Fig. 7b (Iteration 200), the spacing of parallel links along these directions is enlarged. This can be explained by the fact that autonomous links on two parallel routes have to compete for through traffic; since the initial hexagon network is dense (as we can see by comparing it to initial grid and complete networks with regard to connectivity and density), when the traffic is insufficient to support both routes, links on one route have to degenerate while those on the other survive. This finding suggests that link spacing in an urban transportation network is a spontaneously emergent property of network dynamics.

\subsection{Sensitivity tests}

As the values of the model parameters listed in Table 2 are specified, changing these values may lead to different results. Therefore, a sensitivity test is taken by re-running the experiments with different parameter values. Taking Experiment 1 as an example, the measures of resultant network topologies are depicted in Fig. 9 with regard to heterogeneity (to save space, only the flow coefficient out of the three coefficients in the cost function is presented).

Increasing the specified walking speed $\left(v_{0}\right)$ from 0.01 to 1.0 decreases the access cost to the network, thereby increasing the travel demand on the network. Since the "weakest link" criteria is concerned only with the relative travel demand across links, however,

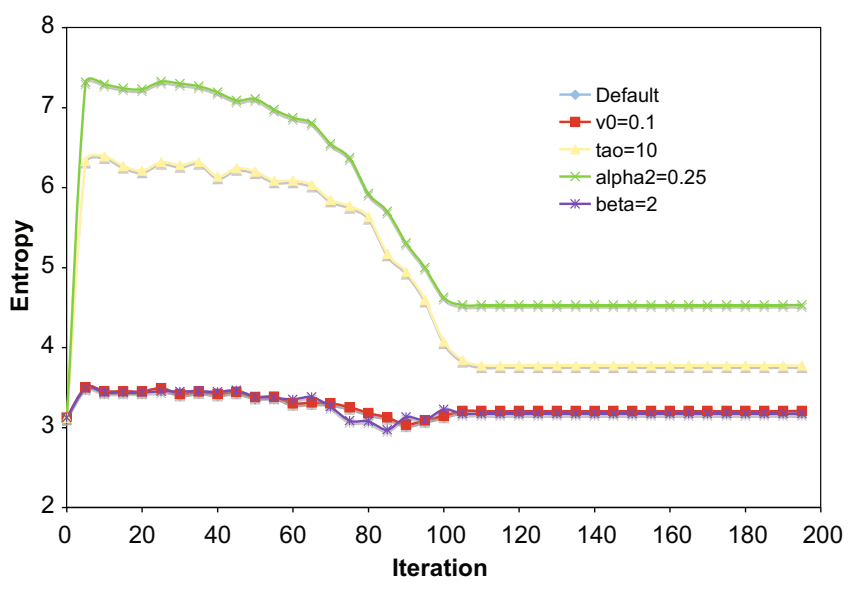

Fig. 9. The sensitivity of topological dynamics on the model parameters with respect to heterogeneity. Each experiment changes the value of one parameter only. The experiments display different fluctuations of entropy.

changing the walking speed did not affect the topological attributes of emergent networks significantly.

A higher toll rate $(\tau)$ means more revenue will be collected on a link with the same amount of through traffic. Raising the toll rate 
from 1.0 to 10.0 significantly increases the average speed of links in equilibrium when the disinvestment starts, therefore resulting in a higher measure of entropy as compared to the default settings. structures.

A lower flow coefficient $\left(\alpha_{2}\right)$ in the cost function indicates a higher economy of volume in maintenance. Decreasing the coefficient from 0.75 to 0.25 favors the links with higher volumes, which essentially intensifies the differentiation of links during the dynamics. This is corroborated by the observation of higher entropy throughout the experiment.

The speed improvement coefficient $(\beta)$ specifies how intensively each individual link invests or disinvests in the infrastructure in response to its profit or deficit. Changing the speed improvement coefficient, however, did not change the link speeds in equilibrium when the disinvestment starts. Therefore, no significant effect on the emergent networks has been observed after increasing this coefficient from 1.0 to 2.0 .

\section{Conclusions}

While fully recognizing that central authorities such as regulators and planners have in recent decades played an active role in transport development, this study tests the evolution of surface transportation networks as a purely spontaneous process which is played out as the outcome of independent decisions made by users and infrastructure suppliers. This research provides an innovative understanding of transport development in terms of demonstrating how decentralized decisions could be translated into facilities on the ground based on myopic local optimal criteria. This research has demonstrated, with both empirical and simulation evidence, independent and sequential decisions that did not follow an optimal design could reproduce the basic features of surface transportation networks. This implies that the formation of surface transportation networks, although different from that of non-spatial "scale-free" networks, could also be self-organized. Additionally, this study contributes to identify and evaluate pre-defined topological features quantitatively and trace their changes in an evolutionary process.

A simulation model is developed to include a process of interaction, investment and disinvestment, thereby enabling a variable topology over time. Meanwhile, the temporal change of topological attributes for networks is evaluated across dimensions of connectivity, density, heterogeneity, and connection patterns. The model is validated using historical data from the Indiana interurban network. Statistical analyses disclose that the model based on myopic investment rules and the intuitive "weakest link" heuristic performs well in predicting the sequence of link abandonment in the interurban network as well as the temporal change of its topological attributes.

The model is then applied on different idealized network structures with different initial conditions. Typical connection patterns such as rings, webs, hub-and-spokes, and cul-de-sacs are observed during network evolution. The fact that the same type of connection patterns may emerge with different initial conditions, or from different network structures, even based on completely decentralized decisions, suggests that surface transportation networks possess robust topological properties that spontaneously emerge from the interaction of demand and supply. Measurement of entropy suggests the spontaneous organization of network hierarchies in a variable network, which agrees with the finding by Yerra and Levinson (2005) and Levinson and Yerra (2006) with fixed network topologies.

The spontaneous change of spacing between parallel links provides further evidence for the interaction between demand and supply during network evolution. The enlargement of spacing is accompanied with the abandonment of links on alternate routes, which is a natural reaction to the over-competition for demand in a dense network. This finding also indicates a future direction of model enhancement to explicitly include the cooperation and competition between decentralized infrastructure suppliers in different ownership structures.

The rise-and-fall of places in terms of their relative importance is also observed as transportation networks evolve, suggesting a coupled evolution of land use and transportation. Following an abundant literature on integrated transport-land use analysis, we have examined the co-evolution of transportation and land use in a parallel study (Levinson, Xie, \& Zhu, 2007).

Due to the complexity our model involves, compromises have to be made between realism and feasibility. In order for an affordable running time, we adopt a simplified four-step travel demand model which neglects congestion effect by assuming infinite link capacity in traffic assignment (it is worthy noting, though, while congestion effect is neglected in the travel demand model, it is indirectly accounted for in the investment models, as congested links generate higher revenue, and therefore get more investment). More sophisticated modeling techniques such as user equilibrium and combined travel demand models would definitely improve model performance by taking into better account congestion and feedback loops between trip distribution and traffic assignment. In fact, one of our ongoing studies on the deployment of transportation networks (Xie \& Levinson, accepted for publication) has introduced user equilibrium travel demand models to produce more realistic traffic estimates.

\section{Acknowledgements}

This research has been supported by the UK Economic and Social Research Council. This material is based upon work supported by the US National Science Foundation under Grant No. 0236396. Any opinions, findings, and conclusions or recommendations expressed in this material are those of the author and do not necessarily reflect the views of the National Science Foundation. We thank three anonymous referees for their constructive comments and suggestions.

\section{References}

Albert, R., Jeong, H., \& Barabasi, A. L. (1999). Diameter of the World-wide Web. Nature, 401, 130-131.

Barabási, A. (2002). Linked: The new science of networks. Perseus Publication.

Barabasi, A. L., \& Albert, R. (1999). Emergence of scaling in random networks. Science, 286, 509.

Barthélemy, M., \& Flammini, A. (2006). Optimal traffic networks. Journal of Statistical Mechanics, L07002.

Christaller, W. (1933). Die zentralen Orte in Sddeutschland. Jena: Gustav Fischer.

Cormen, T. H., Leiserson, C. E., Rivest, R. L., \& Stein, C. (1990). Introduction to algorithms. The MIT Press [chapter 17].

Csányi, G., \& Szendröi, B. (2004). Fractal-small-world Dichotomy in real-world networks. Physical Review E, 70.

de Dios Ortuzar, J., \& Willumsen, L. G. (2001). Modeling transport. John Wiley and Sons, Ltd.

de Solla Price, D. J. (1965). Networks of scientific papers. Science, 149, 510-515.

Dorogovtsev, S., \& Mendes, J. F. F. (2002). Evolution of networks. Advance in Physics, $51,1079$.

Forstall, R. L. (1996). Population of States and Counties of the United States: 17901990. United States Department of Commerce, Bureau of the Census, Population Division.

Garrison, W. L., Marble, D. F. (1962). The structure of transportation networks Technical Report.

Gastner, M. T., \& Newman, M. E. J. (2006). The spatial structure of networks. The European Physical Journal B, 49, 247-252.

Haley R. M. (1936). The American electric railway interurban. PhD thesis, Northwestern University.

Harggett, P., \& Chorley, J. C. (1969). Network analysis in geography. Butler and Tanner Ltd.

Helbing, D., Keltsch, J., \& Molnr, P. (1997). Modeling the evolution of human trail systems. Nature, 388, 47. 
Higgins, J. J. (2003). Introduction to modern nonparametric statistics. Duxbury Press. Hilton, G. W., \& Due, J. F. (1960). The electric interurban railways in America. Stanford: Stanford University Press.

Isard, W. (1956). Location and space-economy. Cambridge: The MIT Press.

Jeong, H., Gombor, B., Albert, R., Oltwai, Z. N., \& Barabási, A. L. (2000). The large-scale organization of metabolic networks. Nature, 407, 651-654.

Jiang, B. (2005). Complex artificial environments, chapter Small world modelling for complex geographic environments. Springer. 259-271.

Jiang, B. (2007). A topological pattern of urban street networks: Universality and peculiarity. Physica A: Statistical Mechanics and its Applications., 384, 647-655.

Jiang, B., \& Claramunt, C. (2004). Topological analysis of urban street networks. Environment and Planning B, 31, 151-162.

Kansky, K. (1969). Structure of transportation networks: Relationships between network geometry and regional characteristics. Chicago: University of Chicago Press.

King, L. (1985). Central place theory. London: SAGE Publication Ltd.

Lam, L., \& Pochy, R. (1993). Active-walker models: Growth and form in nonequilibrium systems. Computation Simulation, 7, 534.

Lämmer, S., Gehlsen, B., \& Helbing, D. (2006). Scaling laws in the spatial structure of urban road networks. Physica A, 363(July), 89-95.

LeBlanc, L. J. (1975). An algorithm for the discrete network design problem. Transportation Science, 9(3), 183-199.

Levinson, D., Xie, F., Zhu, S. (2007). The co-evolution of land use and road networks In Proceedings of the 17th international symposium on transportation and traffic theory (ISTTT).

Levinson, D., \& Yerra, B. (2006). Self organization of surface transportation networks. Transportation Science, 40(2), 179-188.

Liljeros, F., Edling, C. R., Amaral, L. A. N., Stanley, H. E., \& Aberg, Y. (2001). The web of human sexual contacts. Nature, 411, 907-908.
Marlette, J. (1959). Electric railroads of Indiana. Indianapolis: Council for Local History.

Montis, A. D., Barthelemy, M., Chessa, A., Vespignani, A., (2006). The structure of inter-urban traffic: A weighted network analysis. URL: <http://arxiv.org/abs/ physics/0507106>.

Morrill (1965). Migration and the growth of urban settlement. Lund Studies in Geography Series B Human Geography, 26, 65-82.

Newell, G. F. (1980). Traffic flow on transportation networks. Cambridge: MIT Press.

Newman, M. (2003). The structure and function of complex networks. SIAM Review, $45,167-256$

Rodrigue, J., Comtois, C., \& Slack, B. (2006). The geography of transport systems. London: Routledge.

Schweitzer, F., Ebeling, F., Rose, H., \& Weiss, O. (1998). Optimization of road networks using evolutionary strategies. Evolutionary Computation, 419-438.

Shannon, C. E. (1948). A mathematical theory of communication. Bell System Technical Journal, 27, 379-423. and 623-656.

Taaffe, E., Morrill, R. L., \& Gould, P. R. (1963). Transportation expansion in underdeveloped countries: A comparative analysis. Geographical Review, 53(4), 503-529.

Xie, F., Levinson, D. (accepted for publication). Jurisdictional control and network growth. Networks and Spatial Economics.

Xie, F., \& Levinson, D. (2007). Measuring the structure of road networks. Geographical Analysis, 39(3), 336-356.

Yamins, D., Rasmussen, S., \& Fogel, D. (2003). Growing urban roads. Networks and Spatial Economics, 3, 69-85.

Yang, H., \& Bell, M. G. H. (1998). Models and algorithms for road network design: A review and some new developments. Transport Reviews, 18, 257-278.

Yerra, B., \& Levinson, D. (2005). The emergence of hierarchy in transportation networks. Annals of Regional Science, 39(3), 541-553. 